\title{
ALOCAÇÃO DE POLOS POR REALIMENTAÇÃO DE ESTADOS PARA UM SISTEMA DE SUSPENSÃO ATIVA
}

\author{
$\underline{\text { Joacy Mesquita da Silva }}{ }^{1}$; Márcia Lissandra Machado Prado ${ }^{2}$ \\ 1. Bolsista PIBIC/Fapesb, Graduando em Engenharia de Computação, Universidade Estadual de Feira de Santana, e- \\ mail: joacymsilva@gmail.com \\ 2. Orientadora, Departamento de Tecnologia, Universidade Estadual de Feira de Santana, e-mail: \\ $\underline{\text { marcia.lissandra@gmail.com }}$
}

PALAVRAS-CHAVE: Teoria de Controle; Alocação de Polos; Sistema de Suspensão Ativa.

\section{INTRODUÇÃO}

Os sistemas de controle são essenciais em diversos campos da ciência e da engenharia, tais sistemas são utilizados nas mais variadas áreas da indústria (OGATA, 2010), além de veículos espaciais, sistemas robóticos, e operações da indústria que abrangem o controle de temperatura, vazão, pressão, estabilização e direção (NISE, 2009).

O problema de projeto de controladores por realimentação de estados para sistemas lineares invariantes no tempo tem sido extensamente tratado na literatura de controle (DORF; BISHOP, 2009; NISE, 2009; OGATA, 2010). Quando se refere à alocação de polos, o problema consiste em assinalar um ganho de realimentação de estados de forma a alocar todos os polos do sistema em malha fechada no semiplano esquerdo aberto ou em uma região predeterminada do semiplano esquerdo do plano $s$ (PRADO, 2006).

O Sistema de Suspensão Ativa é um exemplo de sistema mecânico utilizado em automóveis e em aplicações industriais. As suspensões veiculares têm por finalidade sustentar, de forma adequada, o chassi do veículo, isolando as vibrações causadas por irregularidades do terreno; manter o contato dos pneus com o solo de modo a manter o carro o mais estável possível; e proporcionar melhor dirigibilidade e conforto aos seus ocupantes (CÔRTE-REAL, 2002).

Este trabalho apresenta o projeto de um controlador por alocação de polos em realimentação de estados, aplicado a um Sistema de Suspensão Ativa.

\section{METODOLOGIA}

Para a pesquisa, foi utilizado o Sistema de Suspensão Ativa da Quanser (QUANSER, 2013). O modelo físico concedido pelo fabricante se encontra na Figura 1.

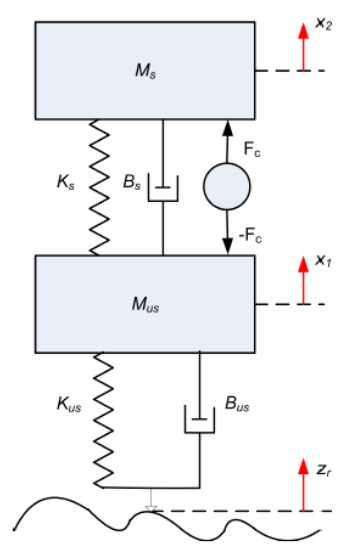

Figura 1: Modelo para o Sistema de Suspensão Ativa da Quanser (QUANSER, 2013). 
O Sistema consiste em duas massas móveis suportadas por um par de molas e amortecedores. A massa superior corresponde ao corpo do veículo suportado acima da suspensão, enquanto a massa inferior se refere a uma das rodas do veículo. A ondulação representa a pista. A modelagem matemática foi possível através da representação do sistema em equações diferenciais, as Equações (1) e (2) foram obtidas.

$$
\begin{gathered}
\frac{d^{2} x_{2}}{d t^{2}}=-g+\frac{F_{c}}{M_{S}}+\frac{B_{s}}{M_{s}} \frac{d x_{1}}{d t}-\frac{B_{s}}{M_{s}} \frac{d x_{2}}{d t}+\frac{K_{s} x_{1}}{M_{S}}-\frac{K_{S} x_{2}}{M_{S}}(1) \\
\frac{d^{2} x_{1}}{d t^{2}}=-g-\frac{F_{c}}{M_{u s}}-\frac{\left(B_{S}+B_{u s}\right)}{M_{u s}} \frac{d x_{1}}{d t}+\frac{B_{u s}}{M_{u s}} \frac{d x_{2}}{d t}+\frac{B_{u s}}{M_{u s}} \frac{d z_{r}}{d t}-\frac{\left(K_{S}+K_{u s}\right) x_{1}}{M_{u s}}+\frac{K_{s} x_{2}}{M_{u s}}+\frac{K_{u s} Z r}{M_{u s}}(2)
\end{gathered}
$$

O sistema possui como entradas a força de controle $F_{c}$ e a perturbação $z_{r}$, que representa as ondulações da pista. A força de controle deve ser capaz de minimizar os efeitos causados pela ação da perturbação. A variável a ser controlada é $x_{2}$, que diz respeito à posição da carroceria do veículo. A variável $x_{1}$ diz respeito à posição do pneu. A aceleração da gravidade é representada pela constante $g$. Os efeitos da força da gravidade podem ser eliminados, considerando pontos de equilíbrio do sistema. A saída considerada, variável a ser controlada, passa a ser chamada de $z_{s}$. Os valores numéricos atribuídos a cada parâmetro da Figura 1 são apresentados na Tabela 1.

Tabela 1. Parâmetros do Sistema de Suspensão Ativa

\begin{tabular}{|c|c|c|c|}
\hline Parâmetro & Identificação & Valor & Unidade \\
\hline$M_{s}$ & Massa do carro & 2,45 & $\mathrm{Kg}$ \\
\hline$M_{u s}$ & Massa do pneu & 1 & $\mathrm{Kg}$ \\
\hline$K_{s}$ & Constante de rigidez da suspensão & 900 & $\mathrm{~N} / \mathrm{m}$ \\
\hline$K_{u s}$ & Constante de rigidez do pneu & 1250 & $\mathrm{~N} / \mathrm{m}$ \\
\hline$B_{S}$ & Coeficiente da suspensão & 7,5 & $\mathrm{~N} . \mathrm{s} / \mathrm{m}$ \\
\hline$B_{u s}$ & Coeficiente do pneu & 5 & $\mathrm{~N} . \mathrm{s} / \mathrm{m}$ \\
\hline
\end{tabular}

Para o Sistema de Suspensão Ativa, foram definidos como vetor de estados, $x$, vetor de entrada, $u$, e vetor de saída, $y$, apresentados nas Equações (3), (4), e (5).

$$
x=\left[\begin{array}{c}
z_{s}-z_{u s} \\
\dot{z_{s}} \\
z_{u s}-z_{r} \\
z_{u s}
\end{array}\right] \text { (3) } u=\left[\begin{array}{l}
\dot{z_{r}} \\
F_{c}
\end{array}\right] \text { (4) } y=\left[\begin{array}{c}
z_{s}-z_{u s} \\
\ddot{z_{s}}
\end{array}\right] \text { (5) }
$$

O primeiro estado representa a deflexão da suspensão, o segundo estado, a velocidade vertical do corpo do veículo, o terceiro estado, a deflexão do pneu, e o quarto estado, a velocidade vertical do pneu (QUANSER, 2013). A primeira entrada para o sistema é a velocidade da superfície da estrada, derivada da perturbação $z_{r}$, a segunda entrada é a força de controle, inicialmente com valor 0 (QUANSER, 2013). A primeira saída do sistema é o curso da suspensão, a segunda saída do sistema é a aceleração do corpo (assumindo que o corpo do veículo é equipado com um acelerômetro) (QUANSER, 2013).

Através das equações diferenciais que representam o sistema, foi possível calcular as matrizes $A, B, C$ e $D$ (DORF; BISHOP, 2009; NISE, 2009; OGATA, 2010). As Equações (6), (7), (8), e (9) trazem essas matrizes. 


$$
\begin{array}{cc}
A=\left[\begin{array}{cccc}
0 & 1 & 0 & -1 \\
-\frac{K_{s}}{M_{s}} & -\frac{B_{s}}{M_{s}} & 0 & \frac{B_{s}}{M_{s}} \\
0 & 0 & 0 & 1 \\
\frac{K_{S}}{M_{u s}} & \frac{B_{S}}{M_{u s}} & -\frac{K_{u s}}{M_{u s}} & -\frac{B_{s}+B_{u s}}{M_{u s}}
\end{array}\right] \text { (6) } & B=\left[\begin{array}{cc}
0 & 0 \\
0 & \frac{1}{M_{s}} \\
-1 & 0 \\
\frac{B_{u s}}{M_{u s}} & -\frac{1}{M_{u s}}
\end{array}\right] \text { (7) } \\
C=\left[\begin{array}{cccc}
1 & 0 & 0 & 0 \\
-\frac{K_{s}}{M_{s}} & -\frac{B_{s}}{M_{s}} & 0 & \frac{B_{s}}{M_{s}}
\end{array}\right](8) \quad D=\left[\begin{array}{ll}
0 & 0 \\
0 & \frac{1}{M_{S}}
\end{array}\right]
\end{array}
$$

Tendo conhecimento das equações de estado e de saída que representam o sistema, além das matrizes $A, B, C$ e $D$, foi possível realizar a construção do diagrama de blocos para efetuar a simulação do sistema no Simulink. Com o intuito do projeto em controlar a posição $z_{S}$ da suspensão, foi necessário o uso de dois integradores na segunda saída para a visualização dessa variável.

Depois de efetuada a modelagem, obtendo as equações de estado e de saída, foi possível simular o comportamento do sistema em malha aberta. Por indicação do fabricante, a perturbação $z_{r}$ é representada por uma onda quadrada de amplitude $0,01 \mathrm{~m}$ e frequência $0,3 \mathrm{~Hz}$. A Figura 2 apresenta a resposta natural do sistema, considerando $z_{r}$ variando de 0 à $0,02 \mathrm{~m}$.

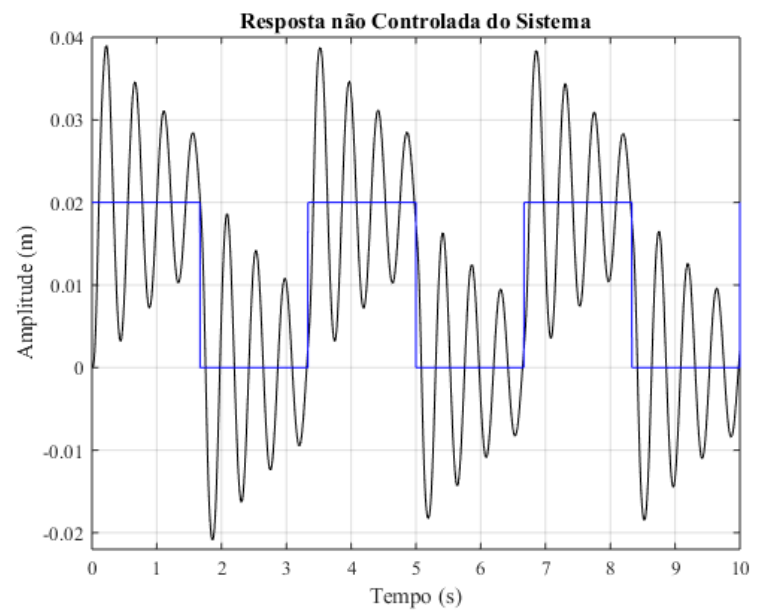

Figura 2: Resposta Natural do Sistema, $z_{r}$ de 0 à $0,02 \mathrm{~m}$.

Através da visualização da Figura 2, pode ser verificada a necessidade de uma ação de controle que minimize as oscilações presentes na suspensão, a fim de promover conforto aos passageiros.

Antes de projetar o controlador, foi necessário especificar requisitos de desempenho para o sistema. Foi adotado que a sobrelevação máxima, $M_{p}$, seria de $5 \%$, e o tempo de estabelecimento, $t_{s}$, seria de 0,5 segundos (DORF; BISHOP, 2009; NISE, 2009; OGATA, 2010).

Baseados na especificação do projeto, foram calculados os valores de $\xi$ e $\omega_{n}$ (baseados no critério de $5 \%$ ), e por consequência foram encontrados os polos reguladores do sistema (DORF; BISHOP, 2009; NISE, 2009; OGATA, 2010).

Para encontrar a matriz de ganho $K$ através da fórmula de Ackermann (OGATA, 2010), foi utilizada a função acker do matlab. Ela recebe como parâmetros as matrizes $A$ e $B$ do sistema, além do vetor contendo os polos desejados para o sistema em malha fechada, e como saída nos traz a matriz de ganho $K$. A Equação (13) apresenta a matriz $K$ encontrada no projeto.

$$
K=\left[\begin{array}{llll}
545,9043 & 38,4895 & 45,0246 & -4,6480
\end{array}\right](13)
$$




\section{RESULTADOS E/OU DISCUSSÃO}

A Figura 2 apresenta a resposta controlada do sistema, e o esforço de controle para alocar os polos nas posições desejadas.
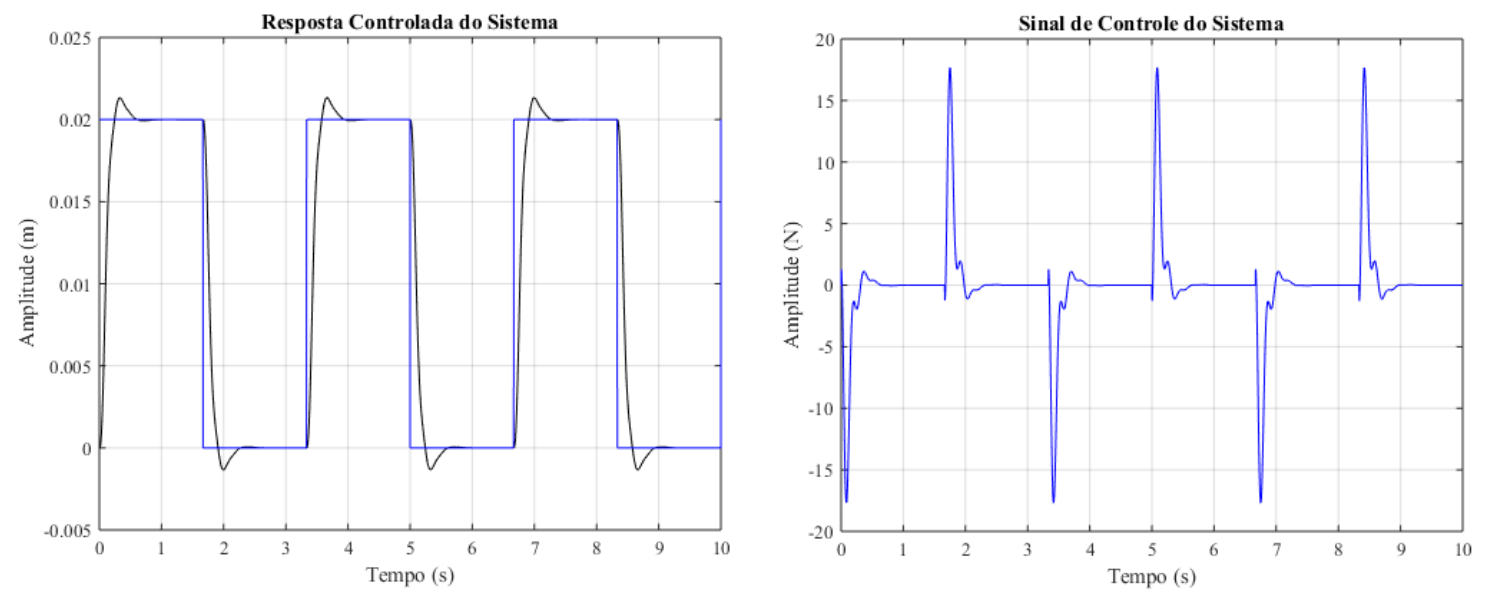

Figura 3: Resposta Controlada e Sinal de Controle do Sistema, $z_{r}$ de 0 à $0,02 \mathrm{~m}$.

A resposta da Figura 3 aponta um $M_{p}$ de aproximadamente 7,5\% e um $t_{s}$ de aproximadamente 0,4 segundos. A ação do controlador nessa simulação apresentou um máximo sinal de controle menor que $20 \mathrm{~N}$, valor que pertence à faixa de $\pm 30 \mathrm{~N}$, que é a faixa limite permitida.

\section{CONCLUSÃO}

Analisando os resultados obtidos com os projetos dos controladores por Alocação de Polos, foi possível perceber que houve o atendimento das especificações do projeto, no que diz respeito à minimização das oscilações do veículo, tempo de estabelecimento e erro de regime nulo.

Como trabalho futuro pode ser citado o projeto de controladores robustos via programação alvo para o Sistema de Suspensão Ativa, considerando o sistema com Realimentação de Estados, utilizando a metodologia proposta por Lordelo e Ferreira (2005). Além disso, poderia se fazer a implementação prática do controlador no sistema real caso se conseguisse obter a planta do sistema.

\section{REFERÊNCIAS}

CÔRTE-REAL, E. W. Dessensibilização da estrutura de controle LQG aplicada ao modelo de uma suspensão ativa utilizando a técnica PRABI. [s.l.] Instituto Militar de Engenharia, 2002.

DORF, R. C.; BISHOP, R. H. Sistemas de Controle Modernos. 11. ed. Rio de Janeiro: LTC, 2009.

NISE, N. S. Engenharia de Sistemas de Controle. 5. ed. Rio de Janeiro: LTC, 2009. OGATA, K. Engenharia de Controle Moderno. 5. ed. São Paulo: Prentice Hall, 2010. PRADO, M. L. M. Controle Robusto por Alocação de Pólos via Análise Intervalar Modal. 2006.

QUANSER. Active Suspension Laboratory Guide. EUA: Quanser, 2013. 